\title{
Clinical and Epidemiological Characteristics of Carbapenem-resistant Klebsiella Pneumoniae Infections in a Tertiary Hospital in China
}

\section{Zhiwen Cui}

Zhengzhou University First Affiliated Hospital https://orcid.org/0000-0002-2118-9928

\section{Lirui Wang}

Zhengzhou University First Affiliated Hospital

\section{Wei Chang}

Zhengzhou University First Affiliated Hospital

\section{Minghui Li}

Zhengzhou University First Affiliated Hospital

\section{Yuexia Li}

Zhengzhou University First Affiliated Hospital

\section{Min Feng ( 451190485@qq.com )}

Zhengzhou University First Affiliated Hospital

\section{Research}

Keywords: Carbapenem-resistant Klebsiella pneumonia, Antimicrobial regimen, Clinical and epidemiological characteristics

Posted Date: January 28th, 2021

DOl: https://doi.org/10.21203/rs.3.rs-153263/v1

License: (c) (1) This work is licensed under a Creative Commons Attribution 4.0 International License. Read Full License 


\section{Abstract}

Background: The infections due to carbapenem-resistant Klebsiella pneumonia (CR-KP) have become an important problem and they are associated with a high mortality rate. The aim of the study is to evaluate the clinical and epidemiological characteristics of CR-KP.

Methods: A retrospective cohort study has been made on all patients presenting with CR-KP infections. 615 patients with CR-KP humor infections diagnosed between January 2018 and December 2019 were identified. 135 patients who did not meet the requirements were excluded, and the remaining 480 patients were enrolled in the study. We have evaluated the mortality in 30 days from the first positive culture. Clinical characteristics, antimicrobial regimens, and outcomes of patients have been analyzed.

Results: The CR-KP infections overall mortality was $37.3 \%$, and bloodstream infections mortality was $66.2 \%$. Survival analysis revealed that there were statistically significant differences between bloodstream infection and pulmonary and drainage fluid infection. The gender, wards, and endotracheal intubation or tracheotomy before positive culture did not differ between the non-survivor and survivor groups. Logistics regression analysis showed that hemopathy, age (>60 years), solid tumors, diabetes, septic shock, acute kidney injury and stroke were independent predictors associated with the 30-day mortality. Multivariate linear regression was performed in APACHE II score, SOFA score, lymphocyte absolute value (LYM) and survival time. Survival time was negatively correlated with APACHE II score and SOFA score, while positively correlated with LYM. In addition, ROC curves were also drawn for APACHE II score, SOFA score and LYM, with AUC of $0.825,0.876$ and 0.797 , respectively. Finally, we investigated different antimicrobial regimens for CR-KP infections. Chi-square test showed that antimicrobial regimen combined carbapenems, tigecycline with polymyxin $B$ was superior the one combined carbapenems with polymyxin $B$, and the difference had statistically significant. But there was not statistically significant difference between carbapenems plus tigecycline and carbapenems plus polymyxin $B$, and it seemed that polymyxin B and tigecycline have synergistic effect. Ceftazidime avibactam-based antimicrobial regimens also had no advantage over other therapeutic regimens.

Conclusions: Our study confirmed there is a high mortality rate in CR-KP infections, especially in the bloodstream infections. The outcome is greatly influenced by the patients' clinical conditions. Antimicrobial regimen combined carbapenems, tigecycline with polymyxin B might be a better choice.

\section{Background}

The carbapenem-resistant Klebsiella pneumonia (CR-KP) infection is a current health threat worldwide [13]. Infections caused by CR-KP isolated are associated with a high mortality rate, ranging from $18 \%$ to $48 \%$, up to $51 \%-72 \%$ in bloodstream infections, depending on the type of therapy administered and different characteristics [4-7].

The first CR-KP was identified in 1993, and since then the CR-KP strains are endemic in many countries [8]. The Annual Report of the European Antibiotic Surveillance Network was published in 2016, reporting a 
mean percentage of carbapenems resistance equal to 6.1\%. In 2018 in China, Klebsiella pneumonia had a resistance rate of $15.4 \%$ to imipenem and $17.9 \%$ to meropenem $[9,10]$.

Although several studies have demonstrated the efficacy of combination regimens in terms of decreased mortality, an effective treatment is still a challenge for clinicians [11-14].

The aim of our study was to evaluate the clinical and epidemiological characteristics of CR-KP infections in a tertiary hospital in China.

\section{Methods}

All patients with CR-KP infection at the First Affiliated Hospital of Zhengzhou University between January 2018 and December 2019 had been identified from the database. The study was approved by the Human Ethics Committee of the First Affiliated Hospital of Zhengzhou University. The patients included in the database were followed by reviewing their medical information and directly contacting the patients.

All patients included in this study have to meet the following criteria: (1) Age between 18 and 80 years; (2) Diagnosis of CR-KP infection was documented by either one or more positive culture; (3) All sputum specimens were bronchial alveolar lavage fluid. Besides, patients who died or discharged within $48 \mathrm{~h}$ after adjusted therapy regimens and patients with incomplete or missing data were excluded.

\section{Patient characteristics}

Patient variables include age, gender, presence of acute or chronic comorbidities, APACHE II score, SOFA score, lymphocyte absolute value (LYM, within 2 days of culture collection), previous surgery ( $\leq 30$ days before culture positive), any invasive procedures ( $\leq 72 \mathrm{~h}$ before culture positive), steroid therapy or immunosuppressive taken over previous 30 days, previous antimicrobial therapy regimens ( $\leq 30$ days), survival days and adjusted therapy regimens after culture positive. Rectal swabs from some patients were collected and screened for the presence of carbapenem-resistant enteric bacteria.

The outcome measured was death within 30 days since the first positive culture. Survivor and nonsurvivor subgroups have been compared to identify predictors of mortality.

\section{Antimicrobial therapies}

The attending physician determined the appropriate initial antimicrobial therapy. Antimicrobial therapy was administered within 5 days after infection onset was defined as empirical therapy. Appropriate definitive antibiotic therapy was defined as that matching the in vitro susceptibility results according to the Clinical and laboratory Standards Institute (CLSI) criteria.

\section{Definitions}

Septic shock is defined as sepsis plus persistent hypotension, requiring vasopressor to maintain mean arterial pressure (MAP) $\geq 65 \mathrm{mmHg}$, and serum lactate levels $>2 \mathrm{mmol} / \mathrm{L}$ despite adequate fluid 
resuscitation.

Acute kidney injury is defined as follows: (1) Increase in serum creatinine by $\geq 0.3 \mathrm{mg} / \mathrm{dl}$ within 48 hours; (2) Increase in serum creatinine by $\geq 1.5$ times baseline; (3) Urine volume $<0.5 \mathrm{ml} / \mathrm{kg} / \mathrm{hour}$ for six hours [16].

Samples of microbial cultures were routinely collected when patients got fever and there was evidence of clinical suspicion or infection. Bloodstream infection was defined as hospital-acquired if the index blood culture had been collected $>48 \mathrm{~h}$ after hospital admission.

\section{Statistical analysis}

Statistical analysis was performed by using SPSS 22.0 (IBM, Armonk, USA). Categorical variables were recorded as absolute numbers and their relative frequencies, and they were compared by the $\chi^{2}$ or Fisher exact test. Categorical variables were analyzed by multivariate logistic regression analysis to identify independent risk factors for mortality. Kaplan-Meier curves were drawn to compare 30-day survival rate in patients with blood stream infection, patients with bronchial alveolar lavage fluid infection and patients with drainage infection. Continuous variables were evaluated by the multiple linear regression analysis. ROC curves were also drawn for APACHE II score, SOFA score and LYM. P value <0.05 was considered statistically significant.

\section{Results}

\section{Baseline characteristics}

We collected 615 patients' clinical data with CR-KP humor infections diagnosed between January 2018 and December 2019. 135 patients who did not meet the requirements were excluded, and the remaining 480 patients were enrolled in the study. Baseline characteristics of study cohort are reported in Table 1.

The total 30-day mortality of patients with CR-KP was $37.3 \%$. Of all patients, the majority were male (289, $60.2 \%)$ and the female were 191 (39.8\%). 379 patients (78.9\%) were pulmonary infection and 68 patients (14.2\%) were bloodstream infection. Hypertension, coronary heart disease, and neurological diseases were the most common underlying diseases (47.7\%, 35.2\% and $21.9 \%$, respectively). Stroke $(188,39.2 \%)$ was the most common in acute complications, followed by AKI (117, 24.4\%) and septic shock (108, $22.5 \%)$, and septic shock was significantly more frequent among patients in the death group than those in the survival group ( $46.3 \%$ vs. $8.3 \%, p<0.001)$. Most of patients $(430,89.6 \%)$ were intubation or tracheotomy and 387 patients (80.6\%) carried a central venous catheter. A proportion of patients got steroid therapy $(93,19.4 \%)$ and continuous renal replacement therapy (CRRT, 58, 12.1\%). Finally, 116 patients' rectal swabs were collected and screened for the presence of carbapenem-resistant enteric bacteria, but only 35 patients were detected positive (30.2\%).

Table 1 Demographic and clinical characteristics of the study cohort 


\begin{tabular}{|c|c|c|c|c|c|}
\hline \multirow[t]{2}{*}{ Variables } & \multicolumn{5}{|c|}{ 30-days outcome } \\
\hline & ALL, 480 & Death, 179 & Survival, 301 & $x^{2}$ & $p$-value \\
\hline \multicolumn{6}{|l|}{ Gender } \\
\hline - Female & 191 & 70 & 121 & 0.06 & 0.813 \\
\hline - Male & 289 & 109 & 180 & & \\
\hline \multicolumn{6}{|l|}{ Age } \\
\hline - $\leq 60$ & 209 & 53 & 166 & 29.51 & $<0.001$ \\
\hline - $>60$ & 271 & 126 & 135 & & \\
\hline \multicolumn{6}{|l|}{ Wards } \\
\hline - Respiratory ICU & 104 & 34 & 70 & 1.2 & 0.273 \\
\hline - General ICU & 110 & 47 & 63 & 1.8 & 0.18 \\
\hline - Surgical ICU & 119 & 48 & 71 & 0.63 & 0.428 \\
\hline - Neurological ICU & 111 & 34 & 77 & 2.74 & 0.098 \\
\hline - Emergency ICU & 36 & 16 & 20 & 0.85 & 0.356 \\
\hline \multicolumn{6}{|l|}{ Specimens } \\
\hline - BALF & 379 & 120 & 259 & 24.41 & $<0.001$ \\
\hline - Blood and catheter tips & 68 & 45 & 23 & 28.27 & $<0.001$ \\
\hline - Drainage liquid & 21 & 7 & 14 & & \\
\hline - Urine & 9 & 1 & 8 & & \\
\hline - Cerebrospinal fluid & 3 & 3 & 0 & & \\
\hline \multicolumn{6}{|l|}{ Comorbidities } \\
\hline - COPD & 85 & 33 & 52 & 0.04 & 0.84 \\
\hline - Solid tumors & 81 & 46 & 35 & 4.01 & 0.045 \\
\hline - Hematopathy & 20 & 16 & 4 & 16.27 & $<0.001$ \\
\hline - Chronic liver disease & 42 & 24 & 18 & 6.85 & 0.009 \\
\hline - Organ transplantation & 20 & 11 & 9 & 2.06 & 0.15 \\
\hline - Coronary heart disease & 169 & 72 & 97 & 2.81 & 0.094 \\
\hline - Hypertension & 229 & 91 & 138 & 0.93 & 0.335 \\
\hline - Diabetes & 97 & 49 & 48 & 8.4 & 0.004 \\
\hline
\end{tabular}




\begin{tabular}{|llllll|}
\hline - Infectious disease & 87 & 36 & 51 & 0.56 & 0.454 \\
\hline - Tuberculosis & 15 & 5 & 10 & 0.10 & 0.747 \\
\hline - CNS sequelae & 105 & 35 & 70 & 0.70 & 0.404 \\
\hline Acute comorbidities & & & & & \\
\hline - Septic shock & 108 & 83 & 25 & 91.09 & $<0.001$ \\
\hline - Acute kidney injure & 117 & 86 & 31 & 84.72 & $<0.001$ \\
\hline - Stroke & 188 & 45 & 143 & 22.64 & $<0.001$ \\
\hline - Acute heart failure & 39 & 17 & 22 & 0.72 & 0.396 \\
\hline Pre-infection variables & & & & & \\
\hline - Central venous catheter & 387 & 159 & 228 & 12.29 & $<0.001$ \\
\hline - Intubation or tracheotomy & 430 & 165 & 265 & 2.06 & 0.151 \\
\hline - CRRT & 58 & 36 & 22 & 17.32 & $<0.001$ \\
\hline - Steroid therapy & 93 & 40 & 53 & 1.61 & 0.2 \\
\hline - Immunosuppressive & 23 & 14 & 9 & 5.74 & 0.017 \\
\hline - Chemoradiotherapy & 19 & 13 & 6 & 8.20 & 0.004 \\
\hline
\end{tabular}

Table 2 Therapies in the death and survive groups 


\begin{tabular}{|llll|}
\hline \multirow{2}{*}{ Variables } & \multicolumn{3}{l}{ 30-days outcome } \\
\cline { 2 - 4 } & ALL & Death & Survive \\
\hline Previous antibiotic therapy & & & \\
\hline -Penicillins \&cephalosporins & 158 & 109 & 166 \\
\hline - Carbapenems & 184 & 69 & 115 \\
\hline - Tigecycline & 69 & 20 & 49 \\
\hline - Quinolones & 153 & 60 & 93 \\
\hline - Others & 63 & 35 & 28 \\
\hline Definitive therapy & & & \\
\hline - Carbapenems & 339 & 150 & 189 \\
\hline - Tigecycline & 327 & 143 & 184 \\
\hline - Polymyxin B & 40 & 27 & 13 \\
\hline - Ceftazidime avibactam & 23 & 14 & 9 \\
\hline - Other cephalosporins & 124 & 36 & 88 \\
\hline - Fosfomycin & 60 & 43 & 17 \\
\hline
\end{tabular}

\section{Therapies}

The empirical and definitive therapies for patients with CR-KP in the survivor and death group are summarized in Table 2. The most frequently used antibiotics for both empirical and definitive therapy were carbapenems in two groups. Then, we did a statistical analysis for the different antibiotic therapeutic regimens and the results were listed in Table 3.

Table 3 Different antibiotic therapeutic regimens 


\begin{tabular}{|lllll|}
\hline \multirow{2}{*}{ Antibiotic therapeutic regimens } & \multicolumn{4}{l}{ 30-days outcome } \\
\cline { 2 - 6 } & ALL & Death & Survive & survival rate \\
\hline A: Ceftazidime avibactam \& Tigecycline & 24 & 7 & 17 & $70.8 \%$ \\
\hline B: Carbapenems \& Tigecycline \& Polymyxin B & 28 & 9 & 19 & $67.9 \%$ \\
\hline C: Carbapenems \& Polymyxin B & 33 & 19 & 14 & $42.4 \%$ \\
\hline D: Carbapenems \& Tigecycline & 198 & 87 & 111 & $56.1 \%$ \\
\hline E: Carbapenems \& Tigecycline \& Fosfomycin & 44 & 18 & 26 & $59.1 \%$ \\
\hline
\end{tabular}

Chi-square test showed that both antibiotic regimen $B$ (carbapenems plus tigecycline and polymyxin $B$, $\chi^{2}=3.96, p=0.04$ ) and antibiotic regimen $A$ (ceftazidime avibactam plus tigecycline, $\chi^{2}=4.52, p=0.033$ ) were superior to antibiotic regimen $C$ (carbapenems combined with polymyxin $B$ ) and the differences were of statistical significance. However, there was no statistically significant difference among antibiotic regimen $D$ (carbapenems plus tigecycline), antibiotic regimen $C$ (carbapenems plus polymyxin $B$ ) and regimen $\mathrm{E}$ (carbapenems plus tigecycline plus fosfomycin). It seemed that polymyxin $\mathrm{B}$ and tigecycline have synergistic effect and carbapenems plus polymyxin B was not a recommended therapeutic regimen (Figure 1). Ceftazidime avibactam-based antimicrobial regimens also had no advantage over other therapeutic regimens.

In addition, we investigated the efficacy of tigecycline combination with other different antibiotics in the treatment of CR-KP. The different antibiotic therapeutic regimens were listed in Table 4.

Table 4 Tigecycline combination with other different antibiotics

\begin{tabular}{|lllllll|}
\hline \multirow{2}{*}{$\begin{array}{l}\text { Tigecycline and } \\
\text { other antibiotics }\end{array}$} & ALL & Death & Survive & Survive rate & $\chi^{2}$ & $p$-value \\
\cline { 2 - 7 } & 97 & 45 & 52 & $53.6 \%$ & 5.432 & 0.246 \\
\hline Imipenem & 39 & 12 & 27 & $69.2 \%$ & & \\
\hline Meropenem & 62 & 30 & 32 & $51.6 \%$ & & \\
\hline Biapenem & 24 & 7 & 17 & $70.8 \%$ & & \\
\hline Ceftazidime avibactam & 39 & 16 & 21 & $56.8 \%$ & & \\
\hline 3rd or 4th ephalosporins & 39 & & & \\
\hline
\end{tabular}

Chi-square test showed that there was no statistically significant difference among different antibiotic regimens. Considering the price of antibiotics, we recommend biapenem combined with tigecycline as a 
therapeutic regimen.

\section{Prognostic factors}

In the multivariate logistic regression analysis, hemopathy, age (>60 years), solid tumors, diabetes, septic shock, acute kidney injury and stroke were independent predictors associated with the 30-day mortality (Table 5).

Table 5 Multivariate analysis of risk factors for mortality

\begin{tabular}{|llll|}
\hline Variables & $\mathrm{B}$ & OR $(95 \% \mathrm{Cl})$ & $p$-value \\
\hline Hemopathy & 2.218 & $9.191(2.555-33.053)$ & 0.001 \\
\hline Age $(>60$ years $)$ & 0.992 & $2.696(1.584-4.590)$ & $<.001$ \\
\hline Solid tumors & 0.835 & $2.305(1.169-4.546)$ & 0.016 \\
\hline Diabetes & 0.677 & $1.968(1.152-3.361)$ & 0.013 \\
\hline Septic shock & 1.796 & $6.024(3.027-11.989)$ & $<0.001$ \\
\hline AKI & 1.218 & $3.381(1.784-6.406)$ & $<0.001$ \\
\hline Stroke & -0.573 & $0.564(0.329-0.966)$ & .037 \\
\hline
\end{tabular}

\section{Correlation analysis and ROC curves}

Multivariate linear regression was performed in APACHE II score, SOFA score, lymphocyte absolute value (LYM) and survival time. Survival time was negatively correlated with APACHE II score and SOFA score, and positively correlated with LYM (Figure 2 and 3). In addition, ROC curves were also drawn for APACHE II score, SOFA score and LYM, with AUC of $0.825,0.876$ and 0.797 , and with cut-off value of 17,6 and 0.775

\section{Survival analysis}

Survival analysis showed that pulmonary and drainage fluid infections were statistically significance differences from bloodstream infection with CR-KP (Figure 4).

\section{Discussion}

In this study we evaluated 30-day mortality in patients with CR-KP infection. The total mortality of patients with CR-KP infection was $37.3 \%$, while with bloodstream infection mortality was $66.2 \%[2,3,5]$. It was patients' characteristics, antibiotic therapeutic regimens as well as immune status of the patients made mortality so high. 
The relatively higher mortality compared with previous studies might be attributable to several factors. First, our hospital is a tertiary referral hospital, and many patients had been very serious when they were admitted to our hospital. The states of the patients were directly related to mortality and had repeatedly reported in previous studies [4, 5]. Second, a previous study has reported that administering the appropriated empirical antibiotic therapy and definitive therapy was an important predictor of patients' outcome $[6,17,18]$. Therefore, it is very important to take the antibiotics reasonably before patients transferred to ICU. Clinical departments, especially surgery, should pay more attention to the rational use of antibiotics. Finally, it is important to detect pathogen and adjust the therapeutic regimen to treat CR-KP infection $[19,24]$. But the positive rate of bacterial culture was low. The positive rate of rectal swabs for the carbapenem-resistant enteric bacteria was only $30.2 \%$ in our study. In recent years, Next Generation Sequencing, which had emerged to help to diagnose CR-KP infection, demand a further study [20].

Independent risk factors associated with mortality were hemopathy, age (>60 years), solid tumors, diabetes, septic shock, acute kidney injury and stroke. Correlation analysis showed that survival time was positively correlated with LYM. Lymphocytes were involved in the patient's innate immunity and adaptive immunity, and low immune status was directly related to patient prognosis [21-23].

We investigated the efficacy of tigecycline combination with different antibiotics (meropenem, imipenem, biapenem, ceftazidime avibactam, and 3rd or 4th ephalosporins) in the treatment of CR-KP, and the differences had not statistically significant. Considering the price of antibiotics, we recommend biapenem combined with tigecycline as a therapeutic regimen. Tigecycline combination with meropenem or ceftazidime avibactam had a higher survival rate. Because there were more neurosurgical patients, meropenem was used more frequently.

Chi-square test showed that both antibiotic regimen 1 (carbapenems plus tigecycline and polymyxin B) and antibiotic regimen 2 (ceftazidime avibactam plus tigecycline) were superior to antibiotic regimen 3 (carbapenems combined with polymyxin B). The reasons may be that polymyxin B was a narrowspectrum antibiotic, and some patients often had multiple drug-resistant bacterial infections. Beside, polymyxin B and tigecycline may have synergistic effect in treating CR-KP infection. Finally, ceftazidime avibactam-based antimicrobial regimens also had no advantage over other therapeutic regimens, but the sample size is too small and it needs further study.

There are some limits in our study. The size of some samples is too small to allow us to detect subtle differences in treatment outcome. Since our data come from a single center, a multi-centers prospective study with more extensive collection of potential confounders is required.

\section{Conclusion}

Our study confirmed a high mortality rate of CR-KP, especially in the bloodstream infections. The outcome is heavily influenced by the patients' clinical conditions. Carbapenems, tigecycline and polymyxin B combined antimicrobial regimen might be a better choice. Ceftazidime avibactam-based antimicrobial 
regimens had no advantage over other therapeutic regimens. Additional data needs to further elucidate this finding especially in light of the introduction of the new agents.

\section{Declarations}

\section{Ethics approval and consent to participate:}

The study was approved by the Human Ethics Committee of the First Affiliated Hospital of Zhengzhou University. Written informed consents were obtained from all patients.

\section{Consent for publication:}

All authors consent for publication.

\section{Availability of data and materials:}

The datasets used and analyzed during the current study are available from the corresponding author on reasonable request.

\section{Competing interests:}

The authors declare that they have no competing interests.

\section{Funding:}

Not applicable.

\section{Authors' contributions:}

ZW C collected data and wrote the initial draft of the manuscript. LR W searched the literatures. W C also collected data and expanded the discussion. $M H L$ was responsible for design and revision of the manuscript. YX L and M F revised the manuscript critically for intellectual content. All authors read and approved the final manuscript.

\section{Acknowledgements:}

Not applicable.

\section{References}

1. Nordmann P, Naas T, Poirel L. Global spread of Carbapenemase-producing Enterobacteriaceae. Emerg Infect Dis. 2011;17(10):1791-8.

2. Tumbarello M, Trecarichi EM, De Rosa FG, et al. Infections caused by KPC-producing Klebsiella pneumoniae: differences in therapy and mortality in a multicentre study. J Antimicrob Chemother. 2015; 70(7):2133-43. 
3. Munoz-Price LS, Poirel L, Bonomo RA, et al. Clinical epidemiology of the global expansion of Klebsiella pneumoniae carbapenemases. Lancet Infect Dis. 2013; 13(9):785-96.

4. Tumbarello $M$, Viale $P$, Viscoli $C$, et al. Predictors of mortality in bloodstream infections caused by Klebsiella pneumoniae carbapenemase-producing K. pneumoniae: importance of combination therapy. Clin Infect Dis. 2012; 55(7):943-50.

5. Daikos GL, Tsaousi S, Tzouvelekis LS, et al. Carbapenemase-producing Klebsiella pneumoniae bloodstream infections: lowering mortality by antibiotic combination schemes and the role of carbapenems. Antimicrob Agents Chemother. 2014; 58(4):2322-8.

6. Qureshi ZA, Paterson DL, Potoski BA, et al. Treatment outcome of bacteremia due to KPC-producing Klebsiella pneumoniae: superiority of combination antimicrobial regimens. Antimicrob Agents Chemother. 2012; 56 (4):2108-13.

7. Fraenkel-Wandel Y, Raveh-Brawer D, Wiener-Well Y, Yinnon AM, Assous MV. Mortality due to blaKPC Klebsiella pneumoniae bacteraemia. J Antimicrob Chemother. 2016; 71(4):1083-7.

8. Falcone M, Russo A, lacovelli A, et al. Predictors of outcome in ICU patients with septic shock caused by Klebsiella pneumoniae carbapenemase-producing K. pneumoniae. Clin Microbiol Infect. 2016; 22(5):444-50.

9. European Centre for Disease Prevention and Control. Surveillance of antimicrobial resistance in Europe 2016. In: Annual report of the European antimicrobial resistance surveillance network (EARSNet). Stockholm: ECDC; 2017.

10. Hu F, Guo Y, Yang Y, et al. Resistance reported from China antimicrobial surveillance network (CHINET) in 2018. Eur J Clin Microbiol Infect Dis. 2019; 38(12):2275-81.

11. Giacobbe DR, Del Bono V, Trecarichi EM, et al. Risk factors for bloodstream infections due to colistinresistant KPC-producing Klebsiella pneumoniae: results from a multicenter case-control-control study. Clin Microbiol Infect. 2015; 21(12):1106.e1-8.

12. Vardakas KZ, Matthaiou DK, Falagas ME, Antypa E, Koteli A, Antoniadou E. Characteristics, risk factors and outcomes of carbapenem-resistant Klebsiella pneumoniae infections in the intensive care unit. J Infect. 2015; 70(6):592-9.

13. Wu HS, Wang FD, Tseng CP, Wu TH, Lin YT, Fung CP. Characteristics of healthcare-associated and community-acquired Klebsiella pneumoniae bacteremia in Taiwan. J Infect. 2012; 64(2):162-8.

14. Machuca I, Gutiérrez-Gutiérrez B, Pérez Cortés S, et al. Oral decontamination with aminoglycosides is associated with lower risk of mortality and infections in high-risk patients colonized with colistinresistant, KPC-producing Klebsiella pneumoniae. J Antimicrob Chemother. 2016; 71(11):3242-9.

15. Seymour CW, Liu VX, Iwashyna TJ, et al. Assessment of Clinical Criteria for Sepsis: For the Third International Consensus Definitions for Sepsis and Septic Shock (Sepsis-3). JAMA. 2016; 315(8):762-74.

16. Ronco C, Bellomo R, Kellum JA. Acute kidney injury. Lancet. 2019; 394(10212):1949-64.

17. Brescini L, Morroni G, Valeriani C, et al. Clinical and epidemiological characteristics of KPC-producing Klebsiella pneumoniae from bloodstream infections in a tertiary referral center in Italy. BMC Infect 
Dis. $2019 ; 19(1): 611$.

18. Qureshi ZA, Paterson DL, Potoski BA, et al. Treatment outcome of bacteremia due to KPC-producing Klebsiella pneumoniae: superiority of combination antimicrobial regimens. Antimicrob Agents Chemother. 2012; 56(4):2108-13.

19. Schechner V, Kotlovsky T, Kazma M, et al. Asymptomatic rectal carriage of blaKPC producing carbapenem-resistant Enterobacteriaceae: who is prone to become clinically infected? Clin Microbiol Infect. 2013; 19(5):451-6.

20. Mendes RE, Jones RN, Woosley LN, Cattoir V, Castanheira M. Application of Next-Generation Sequencing for Characterization of Surveillance and Clinical Trial Isolates: Analysis of the Distribution of $\beta$-lactamase Resistance Genes and Lineage Background in the United States. Open Forum Infect Dis. 2019; 6(Suppl 1):S69-78.

21. Xiong H, Keith JW, Samilo DW, Carter RA, Leiner IM, Pamer EG. Innate Lymphocyte/Ly6C(hi) Monocyte Crosstalk Promotes Klebsiella Pneumoniae Clearance. Cell. 2016; 165(3):679-89.

22. Bengoechea JA, Sa Pessoa J. Klebsiella pneumoniae infection biology: living to counteract host defences. FEMS Microbiol Rev. 2019; 43(2):123-44.

23. Kumar P, Chen K, Kolls JK. Th17 cell based vaccines in mucosal immunity. Curr Opin Immunol. 2013; 25(3):373-80.

24. Guo K, Gong W, Zheng T, et al. Clinical parameters and outcomes of necrotizing soft tissue infections secondary to gastrointestinal fistulas. BMC Infect Dis. 2019; 19(1):597.

\section{Figures}




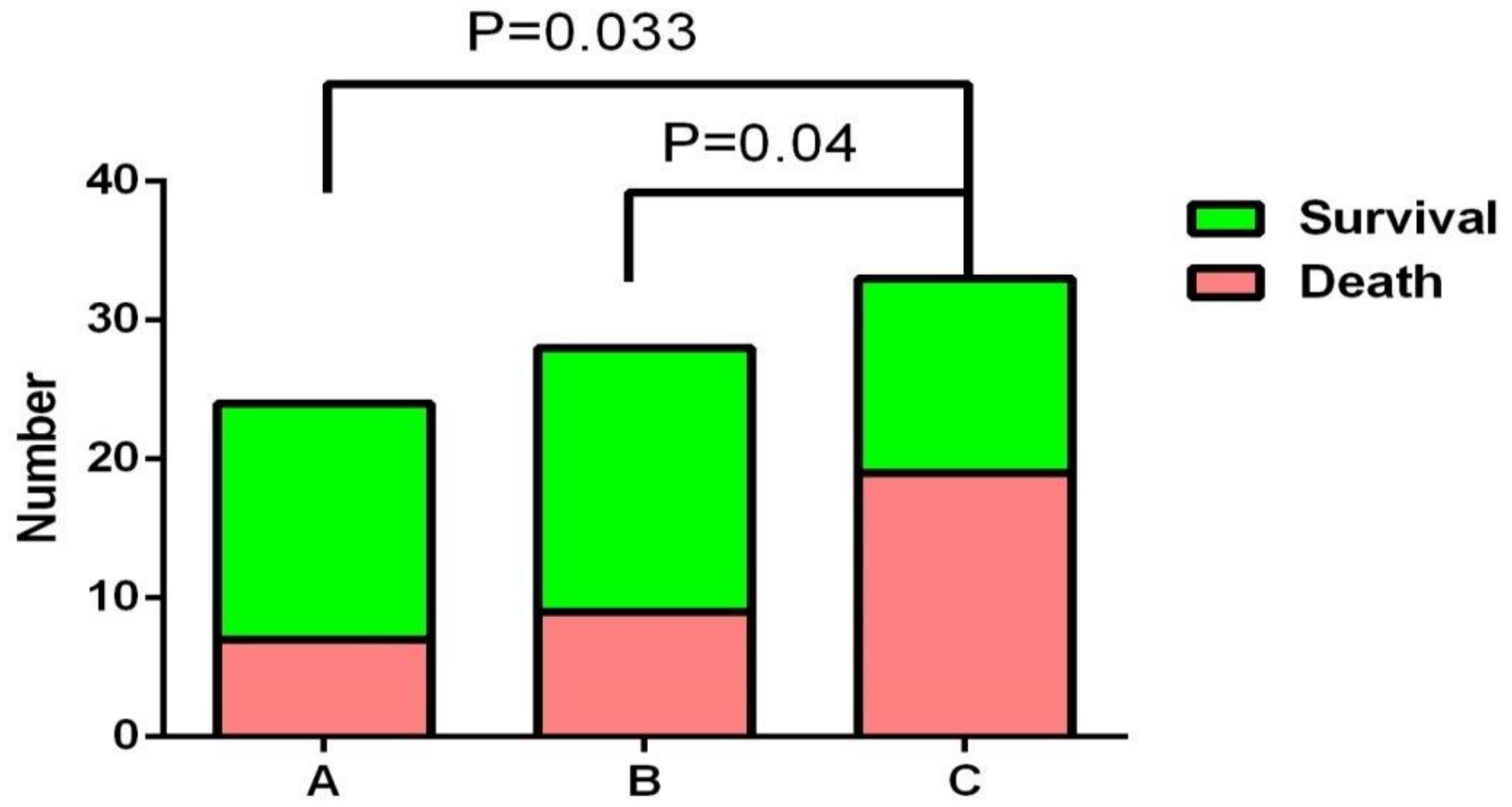

Figure 1

A: Ceftazidime avibactam \& Tigecycline; B: Carbapenems \& Polymyxin B \& Tigecycline; C: Carbapenems \& Polymyxin B. 


\section{Linear regression}

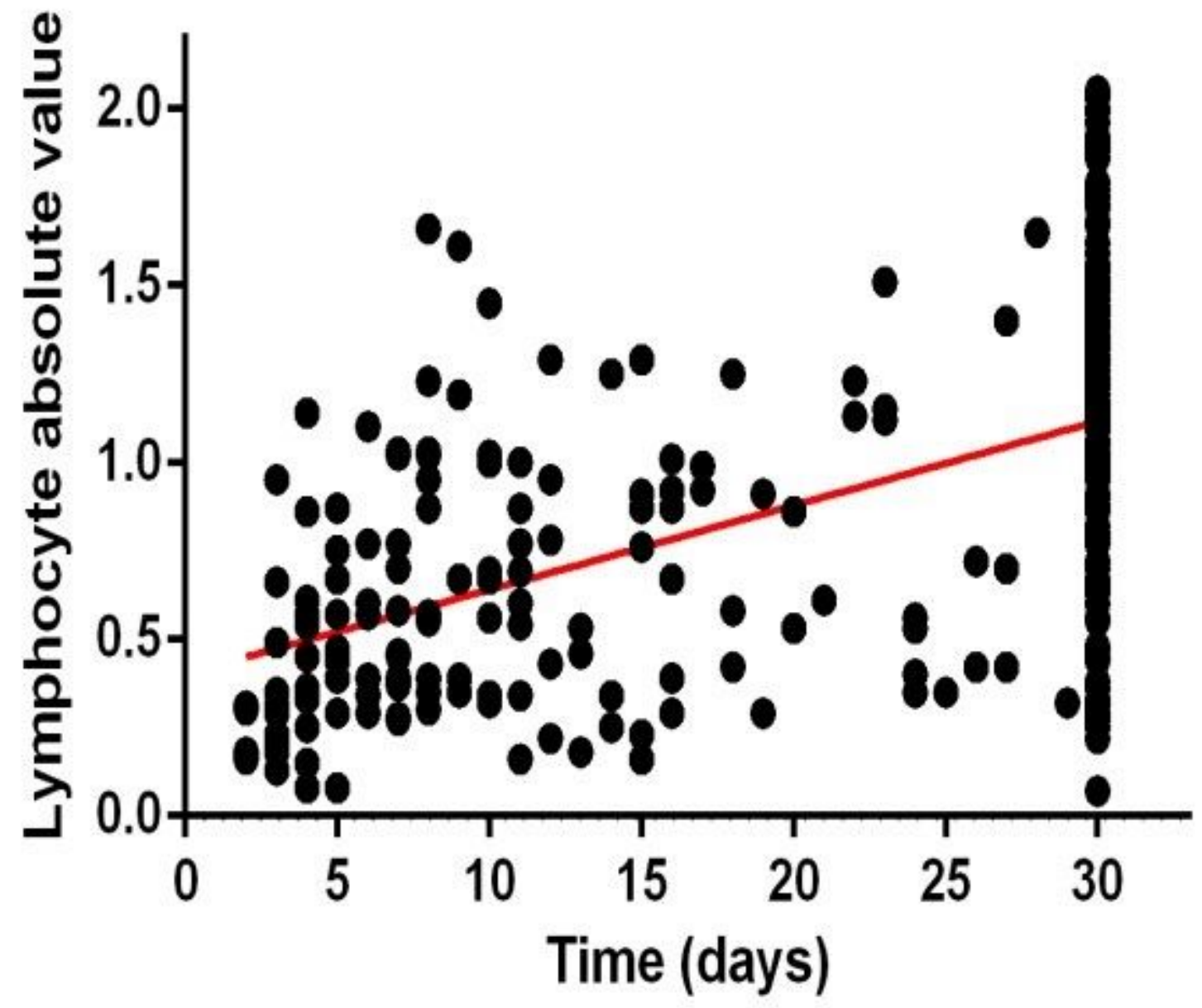

Figure 2

Linear regression analyses for LYM 


\section{Linear regression}

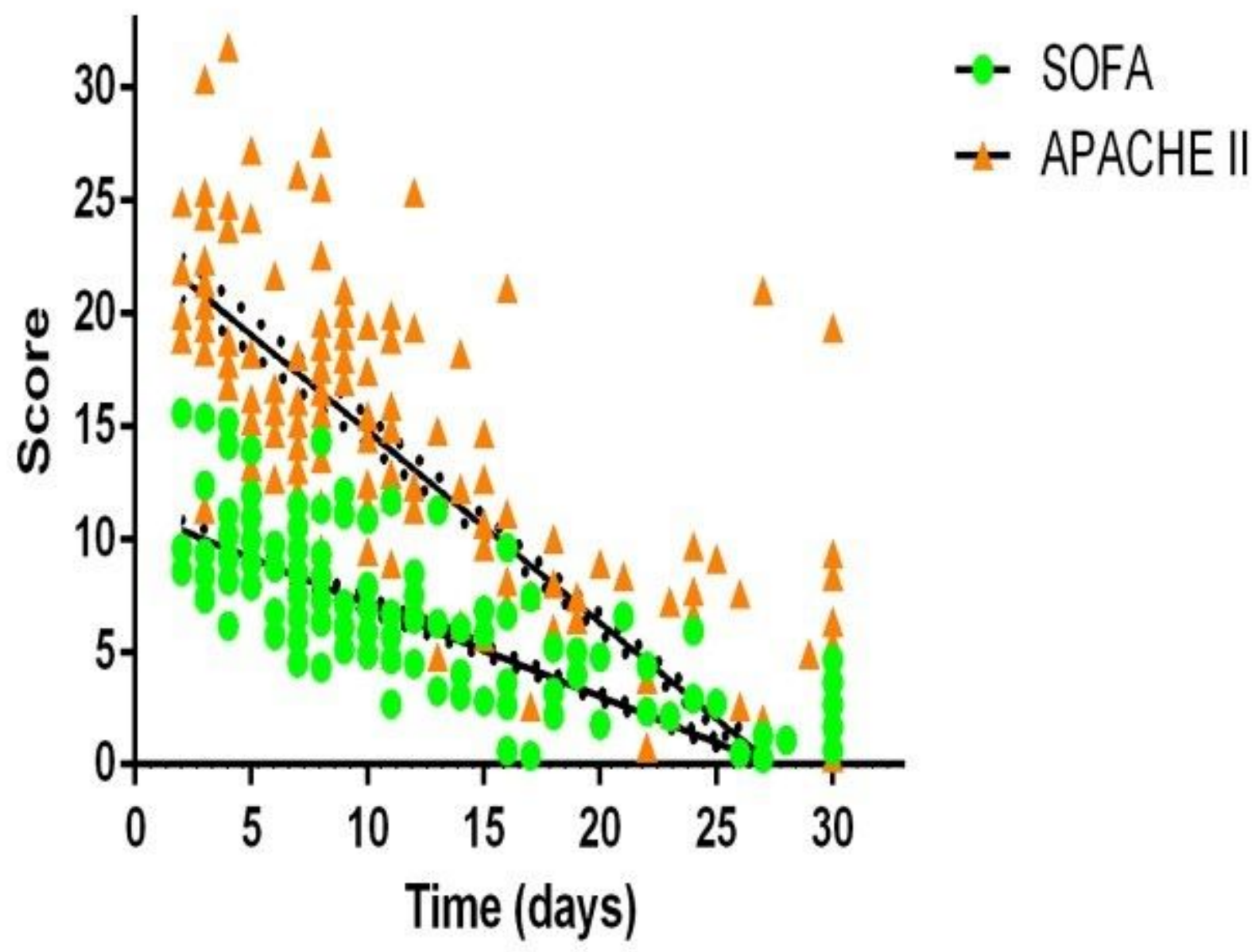

Figure 3

Linear regression analyses for SOFA and APACHE II 


\section{Survival analysis}

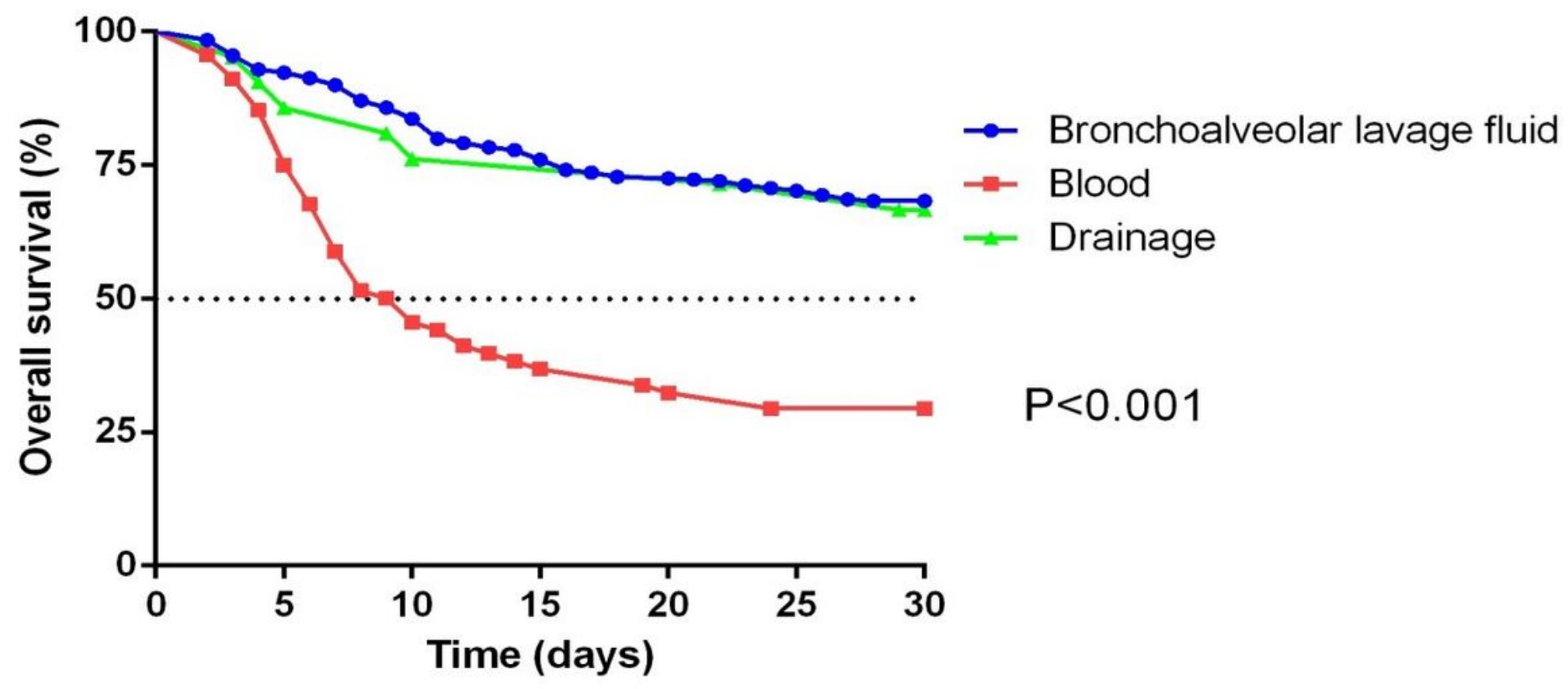

Figure 4

Kaplan Meier survival curves of bloodstream infection, pulmonary and drainage fluid infection. 